\title{
Salvage pembrolizumab added to kinase inhibitor therapy for the treatment of anaplastic thyroid carcinoma
}

Priyanka C. Iyer ${ }^{1}$, Ramona Dadu', Maria Gule-Monroe ${ }^{2}$, Naifa L. Busaidy ${ }^{1}$, Renata Ferrarotto ${ }^{3}$, Mouhammed Amir Habra ${ }^{1}$, Mark Zafereo ${ }^{4}$, Michelle D. Williams ${ }^{5}$, G. Brandon Gunn ${ }^{6}$, Horiana Grosu', Heath D. Skinner ${ }^{6}$, Erich M. Sturgis ${ }^{4}$, Neil Gross ${ }^{4}$ and Maria E. Cabanillas ${ }^{1 *}$

\begin{abstract}
Background: Anaplastic thyroid carcinoma (ATC) is a rare but deadly form of thyroid cancer. Kinase inhibitors kinase inhibitors have shown clinical efficacy in the management of ATC, however, eventually these tumors acquire resistance to $\mathrm{Kl}$ and patients succumb to their disease. Salvage therapy in this setting is limited. As ATC tumors diffusely express the programmed cell death protein ligand (PD-L1), anti- programmed cell death protein (PD-1) drugs such as pembrolizumab offer therapeutic potential. We sought to explore the efficacy of adding pembrolizumab to kinase inhibitors at progression in ATC.
\end{abstract}

Methods: We retrospectively reviewed the charts of ATC patients initiated on pembrolizumab in combination with KI at the time of progression on kinase inhibitors at MD Anderson Cancer Center between August 2016 and August 2017. Efficacy was evaluated with best overall response (BOR) using RECISTV1.1 criteria. Progression free survival (PFS) from the start of pembrolizumab and overall survival (OS) from the start of kinase inhibitors, as well as from the time of addition of pembrolizumab were calculated.

Results: Twelve patients were treated with combination kinase inhibitors plus pembrolizumab at the time of progression on their $\mathrm{KI}$ therapy. Median age at initiation of pembrolizumab was 60 years (range $47-84$ years). BOR was as follows: $5 / 12(42 \%)$ had partial response, 4/12 (33\%) had stable disease and 3/12 (25\%) had progressive disease. Median OS from the start of kinase inhibitor was 10.43 months $(95 \% \mathrm{Cl}=6.02,14.83$, range $5.4-40$ months). Median OS and PFS from the addition of pembrolizumab were 6.93 months $(95 \% \mathrm{Cl}=1.7,12.15$, range $3-15.9$ months) and 2.96 months $(95 \% \mathrm{Cl}=2.2$, 3.7, range 0.57-13.14 months), respectively. Fatigue, anemia and hypertension were the most common AEs encountered on these combinations. Therapy had to be discontinued in 2 patients due to drug induced rash and altered mental status likely from progression of disease.

Conclusion: In a subset of ATC patients, pembrolizumab may be an effective salvage therapy added to kinase inhibitors at the time of progression on these drugs. However, better treatment strategies aimed at incorporating immunotherapy in patients with ATC should be explored. Frontline combination of KI with immunotherapy should be studied in prospective clinical trials.

Keywords: Anaplastic thyroid cancer, Immunotherapy, Salvage, Thyroid cancer, Lenvatinib, Dabrafenib, Trametinib, Undifferentiated

\footnotetext{
* Correspondence: mcabani@mdanderson.org

${ }^{1}$ Department of Endocrine Neoplasia and Hormonal Disorders, The University

of Texas MD Anderson Cancer Center, 1515 Holcombe Blvd, Unit 1461,

Houston, TX 77030, USA

Full list of author information is available at the end of the article
}

(c) The Author(s). 2018 Open Access This article is distributed under the terms of the Creative Commons Attribution 4.0 International License (http://creativecommons.org/licenses/by/4.0/), which permits unrestricted use, distribution, and reproduction in any medium, provided you give appropriate credit to the original author(s) and the source, provide a link to the Creative Commons license, and indicate if changes were made. The Creative Commons Public Domain Dedication waiver (http://creativecommons.org/publicdomain/zero/1.0/) applies to the data made available in this article, unless otherwise stated. 


\section{Background}

Anaplastic thyroid carcinoma (ATC) is rare, but the most aggressive form of thyroid cancer, accounting for fewer than $2 \%$ of all thyroid cancers but responsible for more than a third of deaths due to thyroid cancer [1]. Median overall survival in these patients has reportedly been around 5 months with a 1 year mortality of $80 \%$ [2]. Clinically, these patients present with very rapidly growing, large tumors, often causing compressive symptoms such as dysphagia and stridor.

Current guidelines recommend surgery in cases where the tumor is resectable and chemoradiation for locoregional control of the disease following surgery or when surgery is not feasible [2]. Studies have shown survival benefit in patients who undergo surgery and/or chemoradiation [3-7]. However, many patients with ATC already have advanced disease with distant metastases at the time of initial presentation, wherein the aforementioned treatment options may not be beneficial. Systemic treatment options for ATC patients with distant metastasis have been limited until the recent discovery of several kinase inhibitors (KI) with promising clinical benefit [8-11].

A better understanding of the molecular genomics of this tumor has led to the identification of several driver mutations in ATC $[1,12-14]$ such as BRAF and RAS. These are present in about $25-46 \%$ and $28 \%$ of tumors, respectively $[1,15,16]$. Recently, the FDA has approved the combination of dabrafenib and trametinib for the treatment of $B R A F$ mutated ATC [17]. Lenvatinib is a multikinase inhibitor of VEGFR1-3, FGFR 1-4, PDGFR- $\alpha$, RET and c-kit, approved by the FDA for the treatment of progressive radioiodine refractory differentiated thyroid cancer. Based on encouraging phase 2 results in Japan, the drug is now approved for ATC in that country [9]. In the Unites States, lenvatinib is currently being studied in clinical trials in the ATC population (NCT02657369).

Resistance to $\mathrm{KI}$ is a common problem in ATC and our understanding of mechanisms of resistance is limited [18]. There are limited treatment options for ATC patients whose disease progresses on KI. Immune deactivation of anti- tumoral responses has been suggested to play a role in solid tumors treated with KI $[19,20]$. Several studies have attempted to characterize the type of immune cells and immune checkpoints present in the ATC tumor microenvironment particularly after treatment with multi-modal therapy and in the setting of kinase inhibitors [19, 21-23]. These studies have shown that ATC tumors express the PD-L1 on the tumor surface and that there is diffuse infiltration of the tumor with T-lymphocytes bearing PD-1 receptor [22]. Pembrolizumab is a monoclonal antibody against the PD-1 receptor approved by the FDA in the treatment of several cancers. Preliminary results from a phase 1 study with pembrolizumab in advanced differentiated thyroid cancers which progressed on standard therapies have shown promising results in term of clinical responses and overall survival [24]. In ATC, despite a low tumor mutation burden, a study reported partial responses in 2 out of 4 ATC patients treated with pembrolizumab [25]. However, in a clinical trial comprising of 30 ATC patients treated with single agent spartalizumab (anti-PD1), partial responses were observed in fewer than $20 \%$ of patients [26]. These responses are on the order of to those seen with systemic cytotoxic chemotherapy such as doxorubicin, paclitaxel and gemcitabine where partial responses were observed in $10-20 \%$ of cases [27]. Additionally, in our experience, patients progress rapidly when the $\mathrm{KI}$ is withdrawn. In our case report published recently [28], our patient progressed rapidly when he was taken off dabrafenib and trametinib at the time of post-operative radiation during which only single agent pembrolizumab was continued. However, on reintroducing the KI therapy, his tumor regressed again.

We sought to study the efficacy of adding pembrolizumab as a salvage therapy at progression in order to overcome resistance to $\mathrm{KI}$ in ATC.

\section{Methods \\ Study population}

Under an Institutional Review Board approved protocol, we queried our ATC database for patients who were initiated on treatment with combination pembrolizumab plus KI at the time of progression while on KI between August 2016 and August 2017 and who were followed at The University of Texas MD Anderson Cancer Center. All pathologic diagnoses of ATC were confirmed by a dedicated head and neck pathologist and all radiological images were reviewed by a single radiologist.

\section{Evaluations and definitions}

Molecular testing on the tumor was done as a standard of care using either immunohistochemistry (IHC) and/or next generation sequencing (NGS) at our center (50 gene somatic mutation analysis panel or by Solid Tumor Genomics Assay v1 looking at 134 genes) or by Foundation One. PD-L1 status was determined by immunohistochemistry (IHC) on tumor tissue (clone 22C3, Dako) obtained at initial diagnosis, before initiation of KI. The efficacy of adding pembrolizumab to KI was determined by best overall response (BOR), progression free survival (PFS) and overall survival (OS). RECIST v1.1 was used to evaluate BOR. Clinical benefit was defined as stable disease (SD) plus partial response (PR). PET images, if available, were also reviewed for assessing metabolic response from baseline. PFS was defined as the time elapsed between adding pembrolizumab while on KI and progression or death whichever occurred first. OS was defined as the time elapsed between addition of the 
pembrolizumab to KI and death. Similarly, median OS was also calculated from the start of KI therapy until death. A single radiologist reviewed all cross-sectional images obtained at baseline and during treatment with pembrolizumab plus KI. Adverse events (AEs) were evaluated using Common Terminology Criteria for Adverse Events version 4.0 (CTCAE v.4.0) [29].

\section{Statistical analysis}

The BOR for individual patients was calculated as percent change in the target lesions from baseline and depicted graphically as a waterfall plot. Kaplan Meier curves were used to describe median OS and PFS. Descriptive statistics were used to summarize patient characteristics and AEs. Statistical analyses were performed using SPSS version 22 .

\section{Results}

\section{Study population}

Twelve ATC patients were treated with combination pembrolizumab plus KI at the time of clinical or radiological progression while on KI therapy, and were followed at our institution. The baseline characteristics are summarized in Table 1. Median age at addition of pembrolizumab was 60 years (range 47-84 years). Eight out of $12(67 \%)$ patients were men. At the time of ATC diagnosis 3/12 (25\%) patients were stage IVB and 9/12 (75\%) stage IVC. All of them had either locoregional progression or appearance of new distant metastatic lesions on radiological staging scans while on KI. The site of metastases at addition of pembrolizumab included the lungs in all patients with stage IVC disease. Of these, one also had a cardiac metastasis which grew in size on KI. At the time of initiating pembrolizumab, 6 patients (50\%) had an ECOG of 1, and 4 patients (33\%) had an ECOG of 2. All patients' tumor tissues were tested for the presence of $B R A F$ V600E, either by IHC or NGS-50 gene somatic mutation analysis panel, 6 (50\%) of which harbored a BRAF V600E mutation.

Prior treatment for ATC included surgery in 5 patients (44\%), external beam radiation (EBRT) with or without radiosensitizing chemotherapy in 6 patients $(50 \%)$, and 3 patients (25\%) bridging chemotherapy with nab-paclitaxel with or without carboplatin, while awaiting mutation testing and procurement of the KI therapy. Details of the individual patients' tumor genomics as well as the treatment modalities received have been described in Additional file 1: Table S1 and Table 2, respectively. PD-L1 status on the tumor, either primary or metastatic, was tested in 9 patients $(75 \%)$ and have been described in Table 1.

Six patients (50\%) who harbored the BRAF mutation were treated with a combination of dabrafenib and trametinib, 5 patients (41\%) with lenvatinib and 1 patient (9\%) was treated with single-agent trametinib. Of the 6 patients treated with a combination of dabrafenib and
Table 1 Baseline characteristics of anaplastict thyroid cancer (ATC) patients treated with pembrolizumab added as a salvage therapy to kinase inhibitor therapy

\begin{tabular}{|c|c|}
\hline & $N=12$ \\
\hline Median Age at treatment start, years (range) & $60(47-84)$ \\
\hline \multicolumn{2}{|l|}{ Gender, n (\%) } \\
\hline $\begin{array}{l}\text { Men } \\
\text { Women }\end{array}$ & $\begin{array}{l}8(67) \\
4(33)\end{array}$ \\
\hline \multicolumn{2}{|l|}{ Ethnicity, n (\%) } \\
\hline $\begin{array}{l}\text { Caucasian } \\
\text { Asian } \\
\text { Black }\end{array}$ & $\begin{array}{l}9(75) \\
2(16) \\
1(9)\end{array}$ \\
\hline \multicolumn{2}{|l|}{ Stage at Diagnosis, n (\%) } \\
\hline $\begin{array}{l}\text { IVA } \\
\text { IVB } \\
\text { IVC }\end{array}$ & $\begin{array}{l}0(0) \\
3(25) \\
9(75)\end{array}$ \\
\hline \multicolumn{2}{|l|}{ Pathology, n (\%) } \\
\hline $\begin{array}{l}\text { ATC only } \\
\text { Papillary thyroid cancer }+ \text { ATC } \\
\text { Poorly differentiated thyroid cancer + ATC } \\
\text { Follicular thyroid cancer +ATC }\end{array}$ & $\begin{array}{l}4(33) \\
4(33) \\
3(25) \\
1(9)\end{array}$ \\
\hline $\begin{array}{l}\text { PD-L1 status, n (\%) } \\
\quad<10 \% \\
11-50 \% \\
51-100 \%\end{array}$ & $\begin{array}{l}10(83) \\
2(20) \\
4(40) \\
4(40)\end{array}$ \\
\hline \multicolumn{2}{|l|}{ Performance Status, n (\%) } \\
\hline $\begin{array}{l}\text { ECOG } 0 \\
\text { ECOG } 1 \\
\text { ECOG } 2\end{array}$ & $\begin{array}{l}2(16) \\
6(50) \\
4(33)\end{array}$ \\
\hline \multicolumn{2}{|l|}{ Previous Treatment for ATC, $\mathrm{n}(\%)^{\mathrm{a}}$} \\
\hline $\begin{array}{l}\text { Surgery, n (\%) } \\
\text { Radiation/chemosensitizing, n (\%) } \\
\text { Bridging Chemotherapy, n (\%) }\end{array}$ & $\begin{array}{l}5(41) \\
6(50) \\
3(25)\end{array}$ \\
\hline \multicolumn{2}{|l|}{ Targeted therapy ${ }^{c}, \mathrm{n}(\%)$} \\
\hline $\begin{array}{l}\text { Lenvatinib } \\
\text { Dabrafenib + trametinib } \\
\text { Trametinib alone }\end{array}$ & $\begin{array}{l}5(41) \\
6(50) \\
1(9)\end{array}$ \\
\hline
\end{tabular}

a Patients received more than 1 modality of therapy

${ }^{\mathrm{b}}$ Chemotherapy as a bridging therapy while awaiting targeted therapy ${ }^{\mathrm{C}}$ All patients with BRAFV600E mutations were treated with dabrafenib + trametinib

trametinib, 5 were on dabrafenib $150 \mathrm{mg}$ twice daily plus trametinib $2 \mathrm{mg}$ daily (full doses). The dose of lenvatinib was $24 \mathrm{mg}$ (full dose) in two patients, $20 \mathrm{mg}$ in two patients and $14 \mathrm{mg}$ in one patient. The patient treated with single agent trametinib was started on $2 \mathrm{mg}$ daily.

Median time from the start of KI therapy to addition of pembrolizumab was 9.6 weeks $(95 \% \mathrm{CI}=8.1,11.1$; range 3-105 weeks). In the patients treated with dabrafenib plus trametinib, pembrolizumab was added after a median of 9.6 weeks ( $95 \% \mathrm{CI}=0,20.5$; range 4.9-105 weeks) while in those on lenvatinib, pembrolizumab was added after a median of 8.7 weeks $(95 \% \mathrm{CI}=6.6,10.86$; range 322 weeks), $p$ value $=0.24$. Pembrolizumab was administered intravenously at a dose of $200 \mathrm{mg}$ every 3 weeks. 
Table 2 Patient characteristics: table below summarizes the individual patient characteristics

\begin{tabular}{|c|c|c|c|c|c|c|c|c|c|c|c|c|}
\hline Patient & $\begin{array}{l}\text { Age at start } \\
\text { of } P(y)\end{array}$ & $\begin{array}{l}\text { Stage at } \\
\text { diagn-osis }\end{array}$ & $\begin{array}{l}\text { Prior treatment } \\
\text { before } \mathrm{Kl}\end{array}$ & $\begin{array}{l}\text { PD-L1 } \\
\text { status }\end{array}$ & $\mathrm{KI}$ & $\begin{array}{l}\text { Time on } \mathrm{Kl} \\
\text { alone }(\mathrm{m})\end{array}$ & $\begin{array}{l}\text { Time on } \\
\mathrm{Kl}+\mathrm{P}(\mathrm{m})\end{array}$ & $\begin{array}{l}\text { Change in target } \\
\text { lesions and } \mathrm{BOR} \\
\text { on } \mathrm{KI}+\mathrm{P}\end{array}$ & $\begin{array}{l}\text { PFS On } \\
\mathrm{KI}+\mathrm{P}(\mathrm{m})\end{array}$ & $\begin{array}{l}\text { OS from } \\
\mathrm{KI}(\mathrm{m})\end{array}$ & $\begin{array}{l}\text { OS from } \\
\mathrm{Kl}+\mathrm{P}(\mathrm{m})\end{array}$ & $\begin{array}{l}\text { Alive (A)/ } \\
\text { Deceased (D) }\end{array}$ \\
\hline 1 & 56 & IVC & BC, CXRT & $5 \%$ & DT & 4.3 & 3.1 & $32 \% \mathrm{PD}$ & 0.7 & 7.4 & 3.1 & $D$ \\
\hline 2 & 68 & IVC & $B C$ & $30 \%$ & DT & 9.6 & 3.8 & $21 \%$ PD & 1.5 & 13.4 & 3.8 & $D$ \\
\hline 3 & 57 & IVB & $\mathrm{Sg}, \mathrm{CXRT}$ & $80 \%$ & L & 1.9 & 6.9 & $19 \%$ PD & 1.5 & 8.9 & 6.9 & $\mathrm{D}$ \\
\hline 4 & 58 & IVC & $\mathrm{Sg}, \mathrm{CXRT}$ & $5 \%$ & DT & 26.2 & 13.9 & $-7 \%$ SD & 0.6 & 40.1 & 13.9 & A \\
\hline 5 & 60 & IVC & $B C$ & $50 \%$ & $\mathrm{~L}$ & 5.5 & 15.8 & $-8 \%$ SD & 12.8 & 21.4 & 15.8 & $A$ \\
\hline 6 & 47 & IVB & $\mathrm{Sg}, \mathrm{CXRT}$ & $60 \%$ & DT & 2.4 & 3 & $-14 \%$ SD & 3 & 5.4 & 3 & D \\
\hline 7 & 69 & IVC & CXRT & $\mathrm{n} / \mathrm{A}$ & DT & 2 & 4.1 & $-19 \%$ SD & 3.1 & 6.2 & 4.1 & $D$ \\
\hline 8 & 60 & IVC & $\mathrm{SC}, \mathrm{BC}$ & $>95 \%$ & DT & 1.2 & 6.2 & $-35 \%$ PR & 6.2 & 7.4 & 6.2 & A \\
\hline 9 & 84 & IVC & Sg-Lobectomy & $>10 \%$ & L & 2.1 & 8.3 & $-45 \%$ PR & 8.3 & 10.4 & 8.3 & $\mathrm{D}$ \\
\hline 10 & 57 & IVC & SC, XRT & $\mathrm{n} / \mathrm{A}$ & $\mathrm{L}$ & 2.4 & 16.1 & $-47 \%$ PR & 13.1 & 18.5 & 16.1 & A \\
\hline 11 & 73 & IVC & $\mathrm{Sg}, \mathrm{RAl}$ & $20 \%$ & T & 3.7 & 4.9 & $-48 \% \mathrm{PR}$ & 2.6 & 6.7 & 4.9 & A \\
\hline 12 & 76 & IVB & $\mathrm{Sg}, \mathrm{RAl}$ & $>90 \%$ & L & 1.5 & 5 & $-69 \%$ PR & 5 & 5.8 & 5 & $D$ \\
\hline
\end{tabular}

Sg Surgery, CXRT Chemoradiation, BC Bridging chemotherapy with paclitaxel with or without carboplatin while awaiting Kl, SC Systemic cytotoxic chemotherapy, XRT external beam radiation, $R A$ radioactive iodine, $D T$ dabrafenib+trametinib, $L$ lenvatinib, $T$ trametinib alone, $w$ weeks, $m$ months, $y$ years, $K I$ kinase inhibitor, BOR best overall response, PFS progression-free survival, $O S$ overall survival, $P D$ progressive disease, $S D$ stable disease, $P R$, partial response

\section{Efficacy of combined pembrolizumab and KI therapy}

Median time on combined therapy was 5.6 months (range 2.9-15.8 months). All 12 patients on the combination of pembrolizumab and KI therapy were evaluable for BOR. In the entire cohort, 5/12 (42\%) had PR, 4/12 (33\%) SD and 3/12 (25\%) PD. Overall, clinical benefit (PR + SD) was seen in $9 / 12(75 \%)$ patients. The BOR is shown in the waterfall plot in Fig. 1. These responses were observed irrespective of their PD-L1 status on their tumor. Median time to BOR was 5.86 weeks (range 3-20 weeks). Partial

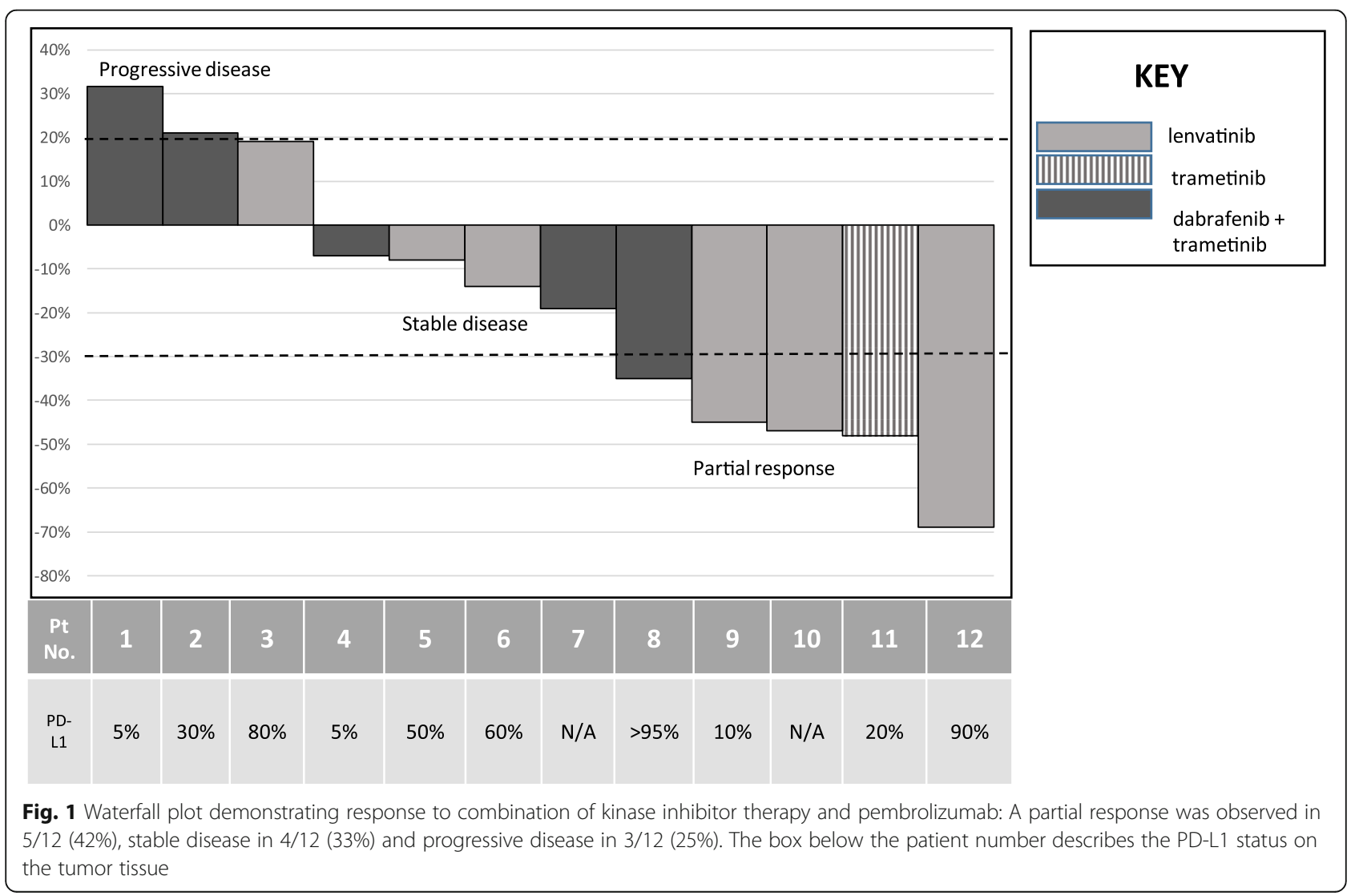


response (PR) was the BOR in 1/6 (17\%) patients on dabrafenib plus trametinib plus pembrolizumab (DTP), 3/5 (60\%) patients on lenvatinib plus pembrolizumab and 1 patient on trametinib plus pembrolizumab. One patient who had a PR with a tumor regression of $47 \%$ on the combination of pembrolizumab and lenvatinib continues to have a response at the time of data analysis. His response to therapy has been shown in Additional file 2: Figure S1.

Stable disease (SD) was the BOR in-4/6 (67\%) patients on dabrafenib, trametinib, pembrolizumab and $1 / 5$ (20\%) patient on lenvatinib plus pembrolizumab (LP). All patients with SD had tumor regression.

PD was the BOR in $-2 / 6(33 \%)$ patients on DTP and $1 / 5(20 \%)$ patient on LP. Both had severe dysphagia from esophageal strictures requiring percutaneous gastrostomy tube placement. These patients were instructed to dilute the capsules in water and administer via gastrostomy tube. One patient was non-compliant with his oral KI which he found too cumbersome.

PETCTs were available in 11/12 patients for assessing response to combination pembrolizumab plus KI. Of these, complete metabolic response was seen in 2 patients at the time of last data analysis.

The timeline of events for each patient from the initiation of combination pembrolizumab plus KI to the date of treatment discontinuation and/or last follow-up are described in Fig. 2.
Median PFS from the addition of pembrolizumab was 2.96 months $(95 \%$ confidence interval $(\mathrm{CI})=2.2,3.7$, range 0.57-13.14 months) for the entire cohort. Patients were continued on the combination of $\mathrm{KI}$ and pembrolizumab at the time of progression.

\section{Survival analysis}

From the start of KI, the median OS was 10.4 months $(95 \%$ $\mathrm{CI}=6.02,14.83$, range 5.4-40 months) for the entire cohort (Fig. 3a). On the basis of type of KI (Fig. 3b), median OS was 7.4 months from the start of dabrafenib plus trametinib (95\% CI $=0.43,14.3$, range 5.4-40 months), 10.4 months from the start of lenvatinib ( $95 \% \mathrm{CI}=7.1,13.8$, range 5.8 21.4 months) and the patient who was started on trametinib was alive 6.7 months after its initiation. From the date of addition of pembrolizumab, the median OS was 6.93 months $(95 \% \mathrm{CI}=1.7,12.15$, range $3-$ 15.9 months) (Fig. 4a) in the entire cohort. The median OS was 3.8 months $(95 \% \mathrm{CI}=2.5,5.1$, range 3 13.9 months) in dabrafenib plus trametinib plus pembrolizumab, 8.25 months $(95 \% \mathrm{CI}=5.4,11.1$, range 516.14 months) in lenvatinib plus pembrolizumab and 4.9 months in trametinib plus pembrolizumab, respectively. (Fig. 4b). After a median follow up of 8.14 months (range 5.75-40.1 months) from the start of KI and 5.6 months (range 2.96-16.14 months) after the addition of pembrolizumab, 5 (42\%) patients were alive.

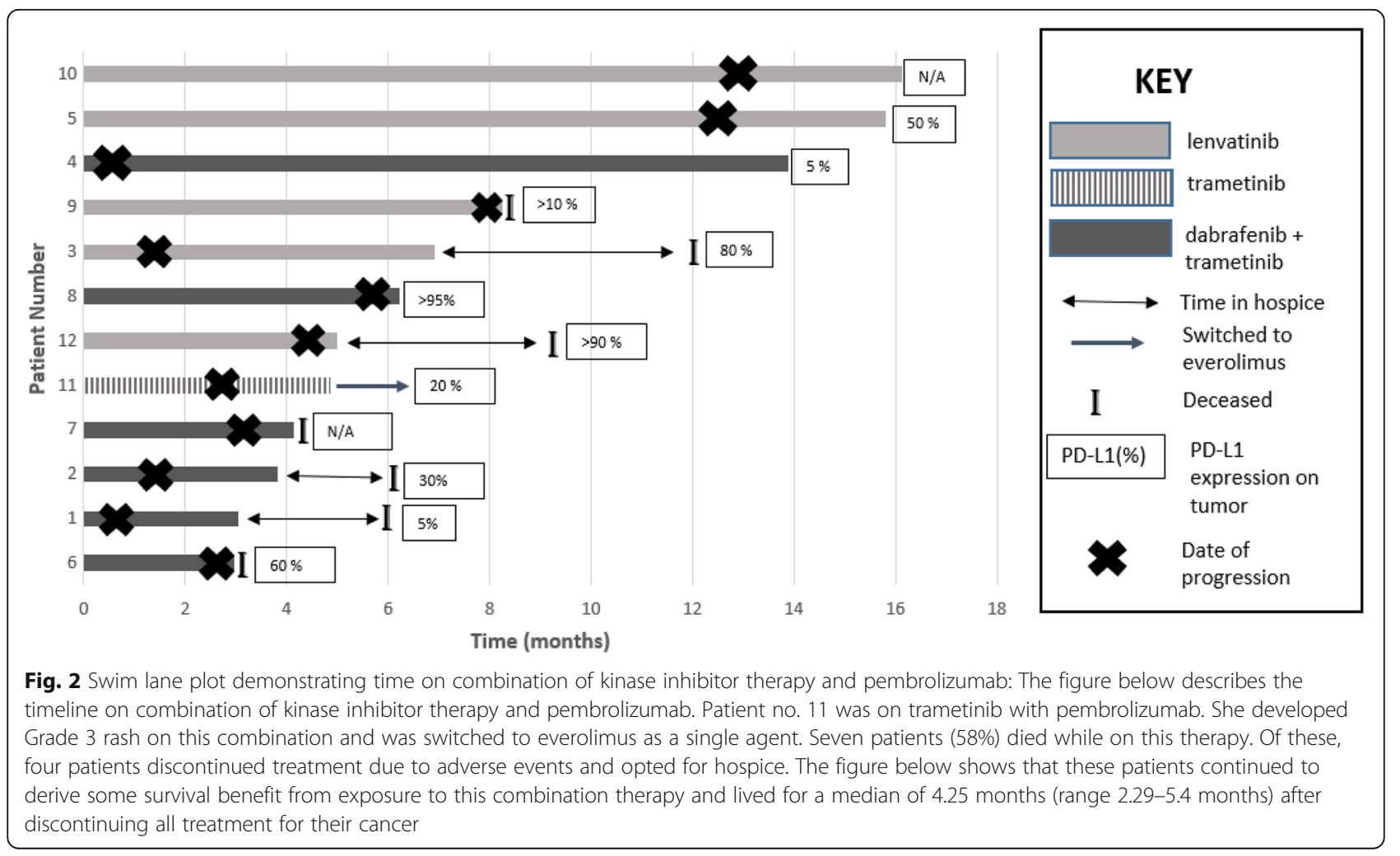




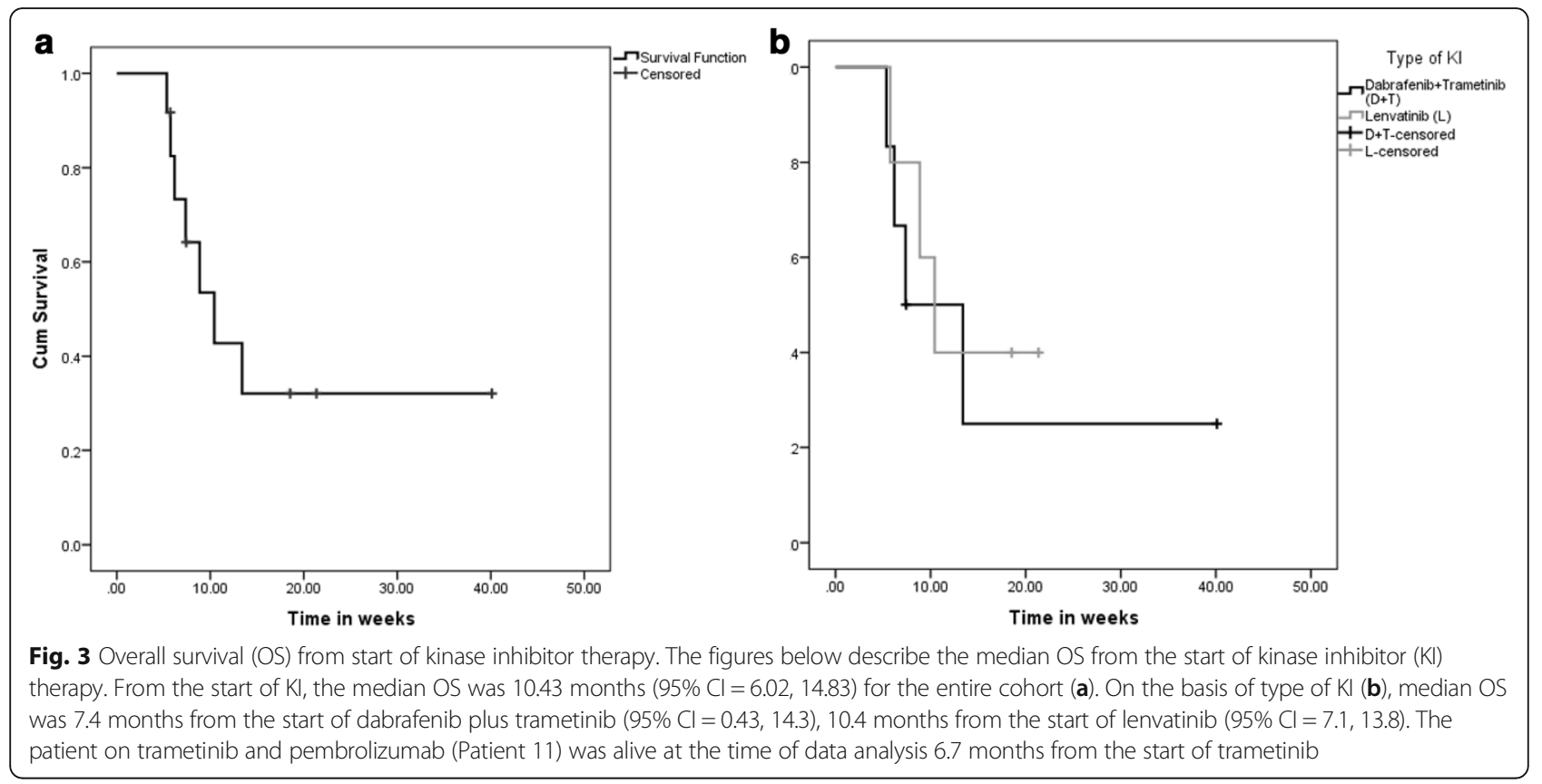

Of the 7 patients who died, 4 patients had opted to discontinue all active treatment and opt for hospice and died a median of 3.3 months (range 2.3-4.2 months) after their last dose of pembrolizumab. Of the 3 patients who died while on combination of pembrolizumab and KI therapy, 1 patient on the combination of DTP died due to bleeding from a tracheo-innominate fistula. It was unclear if this was from tumor shrinkage versus PD. This patient had a history of prior surgeries, tracheostomy and external beam radiation to the neck. The other 2 died of PD.

Of the 5 patients who are alive at the time of data analysis, 2 of them had a complete metabolic response on PET-CT and 1 patient had complete metabolic response in distant metastases with persistent but stable residual avidity in the neck. These three patients have had an OS of over 12 months since the start of pembrolizumab.
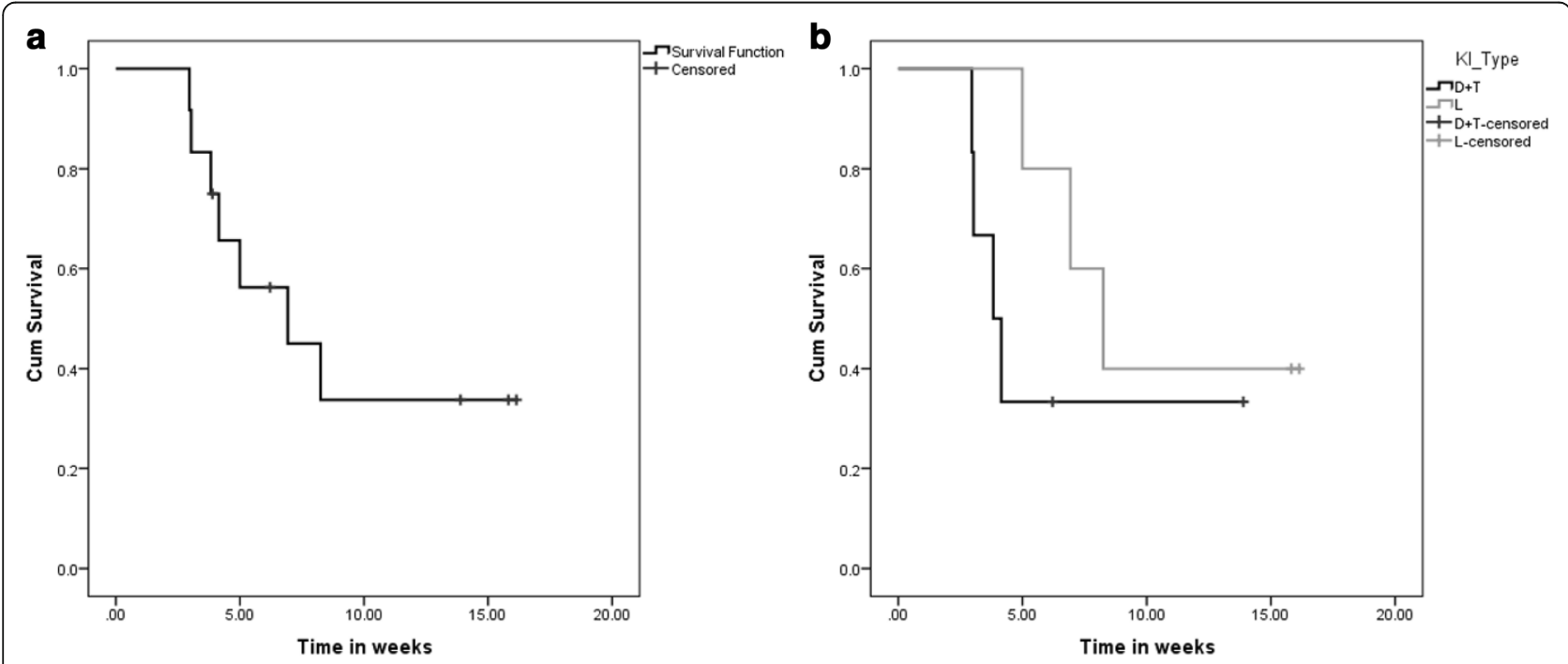

Fig. 4 Overall survival (OS) after the addition of pembrolizumab. The figures below describe the median OS from the start of pembrolizumab added to their respective kinase inhibitor (KI) therapy. From the date of addition of pembrolizumab, the median OS was 6.93 months $(95 \% \mathrm{Cl}=1.7,12.15)(\mathbf{a})$. In the patients who were on dabrafenib plus trametinib while on pembrolizumab, the median OS was 3.8 months $(95 \% \mathrm{Cl}=2.5,5.1)$. Similarly the median OS of the patients who were on the combination of lenvatinib plus pembrolizumab was 8.25 months $(95 \% \mathrm{Cl}=5.4,11.1)$, from the start of pembrolizumab (b). The patient on trametinib is alive 4.9 months after starting pembrolizumab in addition to her trametinib 
Safety and tolerability

AEs were as expected and manageable. All AEs reported have been described in Table 3.

Immune-mediated AEs were seen in 2 patients on combination of lenvatinib and pembrolizumab. Grade 2 colitis was seen in one patient treated with combination of pembrolizumab and lenvatinib and this was treated with budesonide without the need to hold pembrolizumab. Another patient developed grade 2 hepatitis after the second dose of pembrolizumab which required discontinuation of pembrolizumab and treatment with high dose prednisone.

Table 3 Adverse events (AEs). The table below lists the AEs on the combination of kinase inhibitor and pembrolizumab. The denominator used is the number of patients on the combination of lenvatinib (Len) with pembrolizumab, dabrafenib plus trametinib $(D+T)$ with pembrolizumab and the patient on trametinib (tram) with pembrolizumab

\begin{tabular}{|c|c|c|c|c|c|c|c|c|c|c|c|c|}
\hline \multirow[t]{2}{*}{ Adverse event } & \multicolumn{3}{|c|}{ All grades, n (\%) } & \multicolumn{3}{|c|}{ Grade 1, n (\%) } & \multicolumn{3}{|c|}{ Grade 2, n (\%) } & \multicolumn{3}{|c|}{ Grade 3, n (\%) } \\
\hline & $\begin{array}{l}\text { Len } \\
n=5\end{array}$ & $\begin{array}{l}D+T \\
n=6\end{array}$ & $\begin{array}{l}\text { tram } \\
n=1\end{array}$ & $\begin{array}{l}\text { Len } \\
n=5\end{array}$ & $\begin{array}{l}D+T \\
n=6\end{array}$ & $\begin{array}{l}\text { tram } \\
n=1\end{array}$ & $\begin{array}{l}\text { Len } \\
n=5\end{array}$ & $\begin{array}{l}D+T \\
n=6\end{array}$ & $\begin{array}{l}\text { tram } \\
n=1\end{array}$ & $\begin{array}{l}\text { Len } \\
n=5\end{array}$ & $\begin{array}{l}D+T \\
n=6\end{array}$ & $\begin{array}{l}\text { tram } \\
n=1\end{array}$ \\
\hline Fatigue & $5(100)$ & $5(83)$ & $1(100)$ & $1(20)$ & $3(50)$ & $1(100)$ & $3(60)$ & $1(17)$ & $1(100)$ & $1(20)$ & $0(0)$ & $0(0)$ \\
\hline Anemia & $5(100)$ & $4(67)$ & $1(100)$ & $5(100)$ & $2(33)$ & $0(0)$ & $0(0)$ & $2(33)$ & $1(100)$ & $0(0)$ & $0(0)$ & $0(0)$ \\
\hline Hypertension & $5(100)$ & $2(33)$ & $1(100)$ & $3(60)$ & $2(33)$ & $1(100)$ & $2(40)$ & $0(0)$ & $0(0)$ & $0(0)$ & $0(0)$ & $0(0)$ \\
\hline Dry Mouth & $5(100)$ & $2(33)$ & $1(100)$ & $5(100)$ & $2(33)$ & $1(100)$ & $0(0)$ & $0(0)$ & $0(0)$ & $0(0)$ & $0(0)$ & $0(0)$ \\
\hline Anorexia & $5(100)$ & $1(17)$ & $1(100)$ & $3(60)$ & $1(17)$ & $1(100)$ & $2(40)$ & $0(0)$ & $0(0)$ & $0(0)$ & $0(0)$ & $0(0)$ \\
\hline Hoarseness & $5(100)$ & $2(33)$ & $0(0)$ & $5(100)$ & $2(33)$ & $0(0)$ & $0(0)$ & $0(0)$ & $0(0)$ & $0(0)$ & $0(0)$ & $0(0)$ \\
\hline Dehydration & $5(100)$ & $1(17)$ & $0(0)$ & $3(60)$ & $1(17)$ & $0(0)$ & $2(40)$ & $0(0)$ & $0(0)$ & $0(0)$ & $0(0)$ & $0(0)$ \\
\hline Hand-foot skin reaction & $4(80)$ & $3(50)$ & $0(0)$ & $4(80)$ & $3(50)$ & $0(0)$ & $0(0)$ & $0(0)$ & $0(0)$ & $0(0)$ & $0(0)$ & $0(0)$ \\
\hline Rash & $4(80)$ & $2(33)$ & $1(100)$ & $3(60)$ & $2(33)$ & $0(0)$ & $1(20)$ & $0(0)$ & $0(0)$ & $0(0)$ & $0(0)$ & $1(100)$ \\
\hline Pain & $4(80)$ & $2(33)$ & $1(100)$ & $3(60)$ & $1(17)$ & $1(100)$ & $1(20)$ & $1(17)$ & $0(0)$ & $0(0)$ & $0(0)$ & $0(0)$ \\
\hline Hypokalemia & $4(80)$ & $2(33)$ & $0(0)$ & $2(40)$ & $1(17)$ & $0(0)$ & $1(20)$ & $1(17)$ & $0(0)$ & $1(20)$ & $0(0)$ & $0(0)$ \\
\hline Weight loss & $4(80)$ & $1(17)$ & $0(0)$ & $2(40)$ & $0(0)$ & $0(0)$ & $2(40)$ & $1(17)$ & $0(0)$ & $0(0)$ & $0(0)$ & $0(0)$ \\
\hline Diarrhea & $4(80)$ & $1(17)$ & $0(0)$ & $3(60)$ & $1(17)$ & $0(0)$ & $1(20)$ & $0(0)$ & $0(0)$ & $0(0)$ & $0(0)$ & $0(0)$ \\
\hline Hypothyroidism & $4(80)$ & $1(17)$ & $0(0)$ & $4(80)$ & $1(17)$ & $0(0)$ & $0(0)$ & $0(0)$ & $0(0)$ & $0(0)$ & $0(0)$ & $0(0)$ \\
\hline Mucositis & $3(60)$ & $2(33)$ & $1(100)$ & $2(40)$ & $2(33)$ & $1(100)$ & $1(20)$ & $0(0)$ & $1(100)$ & $0(0)$ & $0(0)$ & $0(0)$ \\
\hline Dysphagia & $3(60)$ & $3(50)$ & $0(0)$ & $2(40)$ & $1(17)$ & $1(100)$ & $1(20)$ & $2(33)$ & $0(0)$ & $0(0)$ & $0(0)$ & $0(0)$ \\
\hline Hyponatremia & $3(60)$ & $2(33)$ & $0(0)$ & $3(60)$ & $1(17)$ & $0(0)$ & $0(0)$ & $0(0)$ & $0(0)$ & $0(0)$ & $1(17)$ & $0(0)$ \\
\hline Hyperuricemia & $3(60)$ & $1(17)$ & $0(0)$ & $3(60)$ & $1(17)$ & $0(0)$ & $0(0)$ & $0(0)$ & $0(0)$ & $0(0)$ & $0(0)$ & $0(0)$ \\
\hline Hypomagnesemia & $3(60)$ & $0(0)$ & $0(0)$ & $3(60)$ & $0(0)$ & $0(0)$ & $0(0)$ & $0(0)$ & $0(0)$ & $0(0)$ & $0(0)$ & $0(0)$ \\
\hline Fever & $2(40)$ & $3(50)$ & $0(0)$ & $1(20)$ & $1(17)$ & $0(0)$ & $1(20)$ & $1(17)$ & $0(0)$ & $0(0)$ & $1(17)$ & $0(0)$ \\
\hline Nausea & $2(40)$ & $2(33)$ & $1(100)$ & $1(20)$ & $2(33)$ & $1(100)$ & $1(20)$ & $0(0)$ & $0(0)$ & $0(0)$ & $0(0)$ & $0(0)$ \\
\hline Hair loss & $2(40)$ & $1(17)$ & $1(100)$ & $2(40)$ & $1(17)$ & $1(100)$ & $0(0)$ & $0(0)$ & $0(0)$ & $0(0)$ & $0(0)$ & $0(0)$ \\
\hline Weakness & $2(40)$ & $0(0)$ & $1(100)$ & $1(20)$ & $0(0)$ & $1(100)$ & $1(20)$ & $0(0)$ & $0(0)$ & $1(0)$ & $0(0)$ & $0(0)$ \\
\hline Hyperglycemia & $2(40)$ & $2(33)$ & $0(0)$ & $2(40)$ & $1(17)$ & $0(0)$ & $0(0)$ & $1(17)$ & $0(0)$ & $0(0)$ & $0(0)$ & $0(0)$ \\
\hline Bleeding & $2(40)$ & $1(20)$ & $0(0)$ & $2(40)$ & $0(0)$ & $0(0)$ & $0(0)$ & $0(0)$ & $0(0)$ & $0(0)$ & $0(0)$ & $0(0)$ \\
\hline Hyperbilirubinemia & $2(40)$ & $1(20)$ & $0(0)$ & $1(20)$ & $0(0)$ & $0(0)$ & $1(20)$ & $0(0)$ & $0(0)$ & $0(0)$ & $0(0)$ & $0(0)$ \\
\hline Elevated alkaline phosphatase & $2(40)$ & $1(20)$ & $0(0)$ & $1(20)$ & $0(0)$ & $0(0)$ & $1(20)$ & $0(0)$ & $0(0)$ & $0(0)$ & $0(0)$ & $0(0)$ \\
\hline Shortness of breath & $1(20)$ & $0(0)$ & $1(100)$ & $1(20)$ & $0(0)$ & $1(100)$ & $0(0)$ & $0(0)$ & $0(0)$ & $0(0)$ & $0(0)$ & $0(0)$ \\
\hline Vomiting & $1(20)$ & $1(17)$ & $0(00$ & $0(0)$ & $0(0)$ & $0(0)$ & $1(20)$ & $1(17)$ & $0(0)$ & $0(0)$ & $0(0)$ & $0(0)$ \\
\hline Transaminitis & $1(20)$ & $0(0)$ & $1(100)$ & $0(0)$ & $0(0)$ & $1(100)$ & $1(20)$ & $0(0)$ & $0(0)$ & $0(0)$ & $0(0)$ & $0(0)$ \\
\hline Altered mental status & $1(20)$ & $0(0)$ & $0(0)$ & $0(0)$ & $0(0)$ & $0(0)$ & $0(0)$ & $0(0)$ & $0(0)$ & $1(20)$ & $0(0)$ & $0(0)$ \\
\hline Lymphopenia & $1(20)$ & $0(0)$ & $0(0)$ & $1(20)$ & $0(0)$ & $0(0)$ & $0(0)$ & $0(0)$ & $0(0)$ & $0(0)$ & $0(0)$ & $0(0)$ \\
\hline Hyperkalemia & $1(20)$ & $0(0)$ & $0(0)$ & $1(20)$ & $0(0)$ & $0(0)$ & $0(0)$ & $0(0)$ & $0(0)$ & $0(0)$ & $0(0)$ & $0(0)$ \\
\hline Thrombocytopenia & $1(20)$ & $0(0)$ & $0(0)$ & $1(20)$ & $0(0)$ & $0(0)$ & $0(0)$ & $0(0)$ & $0(0)$ & $0(0)$ & $0(0)$ & $0(0)$ \\
\hline Hypophosphatemia & $1(20)$ & $0(0)$ & $0(0)$ & $0(0)$ & $0(0)$ & $0(0)$ & $0(0)$ & $0(0)$ & $0(0)$ & $1(20)$ & $0(0)$ & $0(0)$ \\
\hline
\end{tabular}




\section{Discussion}

We describe a series of 12 ATC patients who were treated with combination pembrolizumab plus KI as a salvage therapy added at the time of progression on KI therapy.

KI therapies targeting BRAF and MEK as well as those inhibiting VEGFR have shown promise in the management of ATC in the setting of a clinical trial as well as in the real world $[8,9,11,30]$. While the median OS of ATC patients has improved on these therapies, these tumors eventually develop resistance resulting in progression of disease and death $[11,31]$. Hence, there is a need to seek salvage therapies in these patients who progress on KI therapy, or use better combinational strategies upfront. In our study, the median OS was 6.9 months from the addition of pembrolizumab, as a salvage, to KI therapy at the time of progression. Due to the lack of a control arm, wherein patients who progressed on KI were not treated with the addition of pembrolizumab, we are unable to compare and definitively state what would be the effect of not starting pembrolizumab on OS at the time of progression. We did observe that patients who had a near complete or complete metabolic response on PET scans at restaging tended to have a longer OS compared to those who did not. This could suggest that the addition of pembrolizumab reduces the metabolic activity of the tumor. However, our numbers are too small to conclusively state a correlation between metabolic response and OS. This would be best explored in prospective clinical trials. To date, KI, especially BRAFi remains the best treatment option in metastatic ATC.

Several human ATC tissue analyses attempting to characterize the type of immune cells and immune checkpoints present in ATC microenvironment have been performed. These studies showed high PDL1 expression and high frequency of TILs [23]. Similar findings were reported in an ATC mouse model [19]. These data point to a hot immunogenic environment that can be targeted with immunotherapy. However, clinical data using immunotherapy in ATC patients is limited [32]. The combination of immunotherapy and KI has shown promise in the treatment of melanoma [33]. What is being explored currently is whether the combination of immunotherapy and targeted therapy is better tolerated and more efficacious when used simultaneously or sequentially [33]. While the use of anti-PD1 therapies such as pembrolizumab as a single agent needs to be explored in ATC, preclinical studies looking at the combination of $\mathrm{KI}$ and anti-PD1 therapy in ATC have suggested a benefit of using them together [19]. Single agent immunotherapy may not be useful as it takes time to start showing its effect as observed in advanced differentiated thyroid cancer tumors [24]. ATC is a rapidly growing tumor which can progress while awaiting the immunotherapy to take effect.
Research in melanoma has suggested the strategy of introducing anti-PD1 therapy before resistance to BRAF inhibitor therapy is expected to develop [33]. However, this needs to be prospectively explored in ATC. The majority of our patients derived clinical benefit from the combination of pembrolizumab added to their kinase inhibitor therapy at the time of progression on the latter. Although this strategy may provide additional benefit in some patients, prospective studies are needed to explore the timing of incorporating immunotherapy in the treatment of ATC patients. Mechanistic studies have suggested the addition of anti-PD1 therapy around the same time as initiation of KI therapy. In a murine model of ATC, the synergistic effect of combining anti-PD1 immunotherapy with BRAF inhibitor therapy has been shown to produce a significant tumor regression as the tumor cells bearing the $B R A F$ V600E mutation tend to bear a higher expression of PD-L1 [19]. Additionally, the use of a BRAF inhibitor without the use of anti-PD1 inhibitor therapy showed increased expression of PD-L1 on BRAF wild type cells which interacts with the PD-1 on infiltrating T-cells inhibiting the anti-tumor immune response contributing to immune resistance and progression of disease. Similar changes in the tumor immune microenvironment have been shown in melanoma patients after 2 weeks of BRAF inhibitor therapy prior to clinical progression [34]. Therefore, by 9 weeks on dabrafenib and trametinib therapy the tumor microenvironment would be very different from what it was at the time of initiating KI therapy, suggesting maximal benefit obtained if pembrolizumab is added to KI earlier in the course of treatment. In a case report of an ATC patient treated with vemurafenib-(a selective BRAF inhibitor) and nivolumab (an anti-PD1 immunotherapy agent), the patient continued to have a response 20 months after starting nivolumab, which was started within days of starting vemurafenib at the earliest sign of progression [32]. Similarly, VEGF inhibition leads to hypoxia mediated increased expression of PD-L1 in certain tumors and has been proposed to have synergistic benefit when combined with anti-PD1 therapy $[35,36]$. The combination lenvatinib and pembrolizumab has been shown to provide benefit in several solid tumors such as renal cell carcinoma (RCC) and non-small cell carcinoma [37]. We propose that ATC patients may benefit from addition of pembrolizumab to KI earlier in their course of treatment.

A study of 16 ATC patients treated with multimodal treatment reported a lower OS in patients with a PD-L1 expression of $>33 \%$ on their tumor [21]. In our case series, responses to anti-PD1 immunotherapy were seen irrespective of the PD-L1 expression on the tumor.

In terms of tolerability, the combination of kinase inhibitor therapy was associated with some grade 2 and grade 3 AEs which were immune-mediated and managed 
using standard treatment protocols outlined in CTCAE $\mathrm{v}$ 4.0. Drug discontinuation was required in 2 patients due to severe AEs such as grade 3 rash in the patient on trametinib plus pembrolizumab and grade 2 hepatitis in a patient on lenvatinib and pembrolizumab. Hepatitis is a commonly encountered AE when anti-PD1 therapy is combined with VEGFR inhibitors as seen in the clinical trials exploring this combination in RCC [37, 38]. Therefore, while responses with the combination of pembrolizumab and KI therapy are impressive, they are also associated with side effects which patients need to be educated about and for which they require close monitoring.

Limited by the retrospective nature of our study and a small sample size, prospective studies are needed to evaluate the correlation between PD-L1 expression, clinical response and survival in ATC. Considering this was a chart review based study, it is possible that several AEs were either under-reported or not graded uniformly and were subjective on the physician evaluating the AEs.

\section{Conclusion}

Pembrolizumab could be used as a safe and effective salvage therapy to be added to kinase inhibitor therapy at the time of progression. Patients might benefit from the addition of pembrolizumab at the earliest sign of progression or earlier in the course of KI therapy in order to obtain maximum clinical and survival benefit from this combination therapy as the immune microenvironment may be less permissive at the time of progression on KI therapy. This combination should be prospectively explored in clinical trials. A trial exploring combination immunotherapy and targeted therapy is currently open and enrolling (NCT03181100).

\section{Additional files}

Additional file 1: Table S1. This table describes the presence of mutations on the tumor tissue as well as in liquid biopsy of the patients in the series as well as the treatment received and their vital status (DOCX $28 \mathrm{~kb}$ )

Additional file 2: Figure S1. Patient number 10 who had a PR with a tumor regression of $47 \%$ on the combination of pembrolizumab and lenvatinib continues to have a response at the time of data analysis. Axial post contrast $C T$ images through the chest demonstrates right lower lobe pulmonary nodule (red arrow) and pleural based left lower lobe nodularity (yellow arrow) on baseline scan(A) with treatment response on subsequent follow up imaging at 5 months (B) and 8 months (C). (PDF 256 kb)

\section{Funding}

This study was supported in part through The University of Texas MD

Anderson Cancer Center's Cancer Center Support Grant CA16672.

\section{Availability of data and materials}

All data generated or analyzed during this study are included in this published article [and its supplementary information files].

\section{Authors' contributions}

MEC was the primary investigator and contributed to conception and design and gave final approval of the version to be published. PCI was involved in acquisition of the data, analysis and interpretation of the data and drafting the manuscript. RD contributed to the conception and design as well as revised it critically for important intellectual content. MGM analyzed scans at baseline and restaging and recorded tumor measurements to calculate RECISTV1.1 responses. NLB, RF, MAH, MZ, GBG,HG,HDS, EMS, NG were involved in data interpretation and critically reviewing the manuscript. MDW was involved in testing tumor tissue for PD-L1 status when available as well as critically reviewing the manuscript. All authors read and approved the final manuscript.

Ethics approval and consent to participate

Approved under IRB protocol PA13-0246.

Consent for publication

IRB approved waiver of consent.

\section{Competing interests}

Ramona Dadu is on the advisory board of BMS. MEC has received research funding from Eisai and Genentech. NLB has received research funding from Novartis and consulting fees from Eisai. Other authors have no competing interests.

\section{Publisher's Note}

Springer Nature remains neutral with regard to jurisdictional claims in published maps and institutional affiliations.

\section{Author details}

${ }^{1}$ Department of Endocrine Neoplasia and Hormonal Disorders, The University of Texas MD Anderson Cancer Center, 1515 Holcombe Blvd, Unit 1461, Houston, TX 77030, USA. ²Department of Diagnostic Radiology, Division of Diagnostic Imaging, The University of Texas MD Anderson Cancer Center, 1515 Holcombe Blvd, Unit 1482, Houston, TX 77030, USA. ${ }^{3}$ Department of Thoracic/Head and Neck Medical Oncology, Division of Cancer Medicine, The University of Texas MD Anderson Cancer Center, 1515 Holcombe Blvd, Unit 0432, Houston, TX 77030, USA. ${ }^{4}$ Department of Head and Neck Surgery, Division of Surgery, The University of Texas MD Anderson Cancer Center, 1515 Holcombe Blvd, Unit 1445, Houston, TX 77030, USA. ${ }^{5}$ Department of Pathology, Division of Pathology/Lab Medicine, The University of Texas MD Anderson Cancer Center, 1515 Holcombe Blvd, Unit 0085, Houston, TX 77030, USA. ${ }^{6}$ Department of Radiation Oncology, Division of Radiation Oncology, Proton Therapy Center, 1515 Holcombe Blvd, Unit 0097, Houston, TX, USA. ${ }^{7}$ Department of Pulmonary Medicine, Division of Internal Medicine, The University of Texas MD Anderson Cancer Center, 1515 Holcombe Blvd, Unit 1462, Houston, TX 77030, USA.

Received: 1 May 2018 Accepted: 20 June 2018

Published online: 11 July 2018

References

1. Landa I, Ibrahimpasic T, Boucai L, Sinha R, Knauf JA, Shah RH, Dogan S, Ricarte-Filho JC, Krishnamoorthy GP, Xu B, et al. Genomic and transcriptomic hallmarks of poorly differentiated and anaplastic thyroid cancers. J Clin Invest. 2016:126(3):1052-66.

2. Smallridge RC, Copland JA. Anaplastic thyroid carcinoma: pathogenesis and emerging therapies. Clin Oncol-Uk. 2010;22(6):486-97.

3. Prasongsook N, Kumar A, Chintakuntlawar AV, Foote RL, Kasperbauer J, Molina J, Garces Y, Ma D, Neben Wittich MA, Rubin J, et al. Survival in response to multimodal therapy in anaplastic thyroid Cancer. J Clin Endocrinol Metab. 2017;102(12):4506-14.

4. Tennvall J, Lundell G, Wahlberg P, Bergenfelz A, Grimelius L, Akerman M, Hjelm Skog AL, Wallin G. Anaplastic thyroid carcinoma: three protocols combining doxorubicin, hyperfractionated radiotherapy and surgery. Br J Cancer. 2002;86(12):1848-53.

5. Sherman EJ, Lim SH, Ho AL, Ghossein RA, Fury MG, Shaha AR, Rivera M, Lin O, Wolden S, Lee NY, et al. Concurrent doxorubicin and radiotherapy for anaplastic thyroid cancer: a critical re-evaluation including uniform pathologic review. Radiother Oncol. 2011;101(3):425-30.

6. Sugitani I, Hasegawa Y, Sugasawa M, Tori M, Higashiyama T, Miyazaki M, Hosoi H, Orita Y, Kitano H. Super-radical surgery for anaplastic thyroid 
carcinoma: a large cohort study using the anaplastic thyroid carcinoma research consortium of Japan database. Head Neck. 2014;36(3):328-33.

7. Foote RL, Molina JR, Kasperbauer JL, Lloyd RV, Mclver B, Morris JC, Grant CS, Thompson GB, Richards ML, Hay ID, et al. Enhanced survival in locoregionally confined anaplastic thyroid carcinoma: a single-institution experience using aggressive multimodal therapy. Thyroid. 2011;21(1):25-30.

8. Subbiah V, Kreitman RJ, Wainberg ZA, Cho JY, Schellens JHM, Soria JC, Wen PY, Zielinski C, Cabanillas ME, Urbanowitz G, et al. Dabrafenib and Trametinib Treatment in Patients With Locally Advanced or Metastatic BRAF V600-Mutant Anaplastic Thyroid Cancer. J Clin Oncol. 2017;36(1):7-13.

9. Tahara M, Kiyota N, Yamazaki T, Chayahara N, Nakano K, Inagaki L, Toda K, Enokida T, Minami H, Imamura $Y$, et al. Lenvatinib for anaplastic thyroid Cancer. Front Oncol. 2017;7:25

10. Hyman DM, Puzanov I, Subbiah V, Faris JE, Chau I, Blay JY, Wolf J, Raje NS, Diamond EL, Hollebecque A, et al. Vemurafenib in multiple nonmelanoma cancers with BRAF V600 mutations. N Engl J Med. 2015;373(8):726-36.

11. Iyer PC, Dadu R, Ferrarotto R, Busaidy NL, Habra MA, Zafereo M, Gross N, Hess KR, Gule-Monroe M, Williams MD, et al. Real-world experience with targeted therapy for the treatment of anaplastic thyroid carcinoma. Thyroid. 2018;28(1):79-87.

12. Kunstman JW, Juhlin CC, Goh G, Brown TC, Stenman A, Healy JM, Rubinstein JC, Choi M, Kiss N, Nelson-Williams C, et al. Characterization of the mutational landscape of anaplastic thyroid cancer via whole-exome sequencing. Hum Mol Genet. 2015;24(8):2318-29.

13. Latteyer S, Tiedje V, Konig K, Ting S, Heukamp LC, Meder L, Schmid KW, Fuhrer D, Moeller LC. Targeted next-generation sequencing for TP53, RAS, BRAF, ALK and NF1 mutations in anaplastic thyroid cancer. Endocrine. 2016;54(3):733-41.

14. Forbes SA, Beare D, Gunasekaran P, Leung K, Bindal N, Boutselakis $H_{4}$ Ding M, Bamford S, Cole C, Ward S, et al. COSMIC: exploring the world's knowledge of somatic mutations in human cancer. Nucleic Acids Res. 2015:43(Database issue):D805-11

15. Cabanillas ME, Busaidy NL, Khan SA, Gunn BG, R D SNR, SG W. Molecular diagnostics and anaplastic thyroid carcinoma: the time has come to harvest the high hanging fruit. IntlJ Endocrine Oncology. 2016;3(3):221-33.

16. Cabanillas ME, Zafereo M, Gunn GB, Ferrarotto R. Anaplastic thyroid carcinoma: treatment in the age of molecular targeted therapy. J Oncol Pract. 2016;12(6):511-8

17. https://www.fda.gov/Drugs/InformationOnDrugs/ApprovedDrugs/ ucm606708.htm. Accessed 05 June 2018.

18. Danysh BP, Rieger EY, Sinha DK, Evers CV, Cote GJ, Cabanillas ME, Hofmann MC. Long-term vemurafenib treatment drives inhibitor resistance through a spontaneous KRAS G12D mutation in a BRAF V600E papillary thyroid carcinoma model. Oncotarget. 2016;7(21):30907-23

19. Brauner E, Gunda V, Vanden Borre P, Zurakowski D, Kim YS, Dennett KV, Amin S, Freeman GJ, Parangi S. Combining BRAF inhibitor and anti PD-L1 antibody dramatically improves tumor regression and anti tumor immunity in an immunocompetent murine model of anaplastic thyroid cancer. Oncotarget. 2016;7(13):17194-211

20. Shin SJ, Jeon YK, Cho YM, Lee JL, Chung DH, Park JY, Go H. The association between PD-L1 expression and the clinical outcomes to vascular endothelial growth factor-targeted therapy in patients with metastatic clear cell renal cell carcinoma. Oncologist. 2015;20(11):1253-60.

21. Chintakuntlawar AV, Rumilla KM, Smith CY, Jenkins SM, Foote RL, Kasperbauer لـ Morris JC, Ryder M, Alsidawi S, Hilger C, et al. Expression of PD-1 and PD-L1 in anaplastic thyroid Cancer patients treated with multimodal therapy: results from a retrospective study. J Clin Endocrinol Metab. 2017;102(6):1943-50.

22. Bastman JJ, Serracino HS, Zhu Y, Koenig MR, Mateescu V, Sams SB, Davies KD Raeburn CD, McIntyre RC Jr, Haugen BR, et al. Tumor-infiltrating T cells and the PD-1 checkpoint pathway in advanced differentiated and anaplastic thyroid Cancer. J Clin Endocrinol Metab. 2016;101(7):2863-73.

23. Dadu R, Rodriguez Canales J, Wistuba I, Tian W, Lui H, Williams MD, ME C. Targeting immune system in anaplastic thyroid cancer (ATC): a potential treatment approach. Thyroid. 2015;25(Supplement 1):749

24. Mehnert JMVA, Brose M, Aggarwal R, Lin C-C, Prawira A, de Braud F, Tamura K, Doi T, Piha-Paul S, Gilbert J, Saraf S, Thanigaimani P, Cheng J, Keam B. Pembrolizumab for advanced papillary or follicular thyroid Cancer: preliminary results from the phase 1b KEYNOTE-028 study. In: American Society of Clinical oncology (ASCO) annual meeting: June 3-7, 2016. Chicago; J Clin Oncol. 2016;34:(suppl; abstr 6091).

25. KP KSA, Mobley B, Burks T, Beg MS, Ross JS, Ali SM, Bowles DW. Relationship of anaplastic thyroid cancer high tumor mutation burden and MSI-H status with response to anti-PD1 monotherapy. In: ASCO. vol. 36, 2018 (suppl; abstr e18114). Chicago: J Clin Oncol; 2018.

26. Wirth LJEE, Capdevila J, Paz-Ares LG, Lin C, Taylor MH, Ramlau R, Butler M, Delord JP, Horvath Z, Gelderblom H, Ascierto PA, Fasolo A, Führer D, Wu H, Bostel G, Cameron S, Faris JE, Varga Al. Phase I/II study of spartalizumab (PDR001), an anti-PD1 $\mathrm{mAb}$, in patients with anaplastic thyroid cancer. J Clin Oncol. 2018;36(suppl; abstr 6024).

27. Denaro N, Nigro CL, Russi EG, Merlano MC. The role of chemotherapy and latest emerging target therapies in anaplastic thyroid cancer. OncoTargets and therapy. 2013;9:1231-41.

28. Cabanillas MEFR, Garden A, Ahmed S, Busaidy NL, Dadu R, Williams MD, Skinner H, Gunn B, Grosu HB, lyer PC, Hofmann MC, Zafereo M. Neoadjuvant biological-based therapy for anaplastic thyroid carcinoma. Thyroid. 2018.

29. Carhill AA, Cabanillas ME, Jimenez C, Waguespack SG, Habra MA, Hu M, Ying A, Vassilopoulou-Sellin R, Gagel RF, Sherman SI, et al. The noninvestigational use of tyrosine kinase inhibitors in thyroid cancer: establishing a standard for patient safety and monitoring. J Clin Endocrinol Metab. 2013:98(1):31-42.

30. Iniguez-Ariza NM, Ryder MM, Hilger CR, Bible KC. Salvage Lenvatinib therapy in metastatic anaplastic thyroid Cancer. Thyroid. 2017;27(7):923-7.

31. Haugen BR, Alexander EK, Bible KC, Doherty GM, Mandel SJ, Nikiforov YE, Pacini F, Randolph GW, Sawka AM, Schlumberger M, et al. 2015 American Thyroid Association management guidelines for adult patients with thyroid nodules and differentiated thyroid Cancer: the American Thyroid Association guidelines task force on thyroid nodules and differentiated thyroid Cancer. Thyroid. 2016;26(1):1-133.

32. Kollipara R, Schneider B, Radovich M, Babu S, Kiel PJ. Exceptional response with immunotherapy in a patient with anaplastic thyroid Cancer. Oncologist. 2017;22(10):1149-51.

33. Atkins MB, Larkin J. Immunotherapy combined or sequenced with targeted therapy in the treatment of solid tumors: current perspectives. J Natl Cancer Inst. 2016;108(6):djv414.

34. Szczepaniak Sloane RA, Gopalakrishnan V, Reddy SM, Zhang X, Reuben A, Wargo JA. Interaction of molecular alterations with immune response in melanoma. Cancer. 2017;123(S11):2130-42.

35. Liu KG, Gupta S, Goel S. Immunotherapy: incorporation in the evolving paradigm of renal cancer management and future prospects. Oncotarget. 2017:8(10):17313-27

36. Barsoum IB, Smallwood CA, Siemens DR, Graham CH. A mechanism of hypoxia-mediated escape from adaptive immunity in cancer cells. Cancer Res. 2014;74(3):665-74

37. Taylor MDC, Schmidt E, Bagulho T, Li D, Shumaker R, Rasco D. 776PD—A phase $1 \mathrm{~b}$ trial of lenvatinib (LEN) plus pembrolizumab (PEM) in patients with selected solid tumors. Ann Oncol. 2016;27(6):266-95.

38. Amin A, Plimack ER, Infante JR, Ernstoff MS, Rini BI, DF MD, Knox JJ, Pal SK, Voss MH, Sharma P, et al. Nivolumab (anti-PD-1; BMS-936558, ONO-4538) in combination with sunitinib or pazopanib in patients (pts) with metastatic renal cell carcinoma (mRCC). J Clin Oncol. 2014;32(15_suppl):5010.

\section{Ready to submit your research? Choose BMC and benefit from:}

- fast, convenient online submission

- thorough peer review by experienced researchers in your field

- rapid publication on acceptance

- support for research data, including large and complex data types

- gold Open Access which fosters wider collaboration and increased citations

- maximum visibility for your research: over $100 \mathrm{M}$ website views per year

At BMC, research is always in progress.

Learn more biomedcentral.com/submissions 